\title{
On Finding Geodesic Equation of Two Parameters Lognormal Distribution
}

\author{
William W.S. Chen ${ }^{1}$ \\ Department Of Statistics, The George Washington University, Washington D.C. 20013
}

\begin{abstract}
In the present paper, we used two different algorithms to solve some partial differential equations, where these equations originated from the well-known two parameters of lognormal distributions. The first approach that we adopted was the classical method which involved solving a triply of partial differential equations. The second approach that we used was the well-known Darboux Theorem. As we expected the two results were identical.
\end{abstract}

\section{Mathematical Subject Classification: $62 \mathrm{e} 99$}

Keywords and Phases: Darboux Theory, Differential Geometry, Geodesic Equation, Partial Differential Equation, Lognormal Distribution.

\section{INTRODUCTION}

The shortest distance between two points on an arbitrary surface has interested both mathematicians and statisticians for a very long time. Mathematicians are usually interested in the following types of mapping: conformal mapping where the angles are preserved isometric mapping where both distances and angles are preserved equiareal mapping where areas are preserved; and geodesic mapping in which geodesics are preserved. While distance function makes statistician useful can be explained from both a theoretical and a practical view. Almost all statistical distributions now in application have their Gaussian Curvature different from zero. For example, Chen[1] has determined that normal distribution has -0.5 while Cauchy distribution equal -2 . This means the shortest distance between the two points is not a straight line but some curvature line. So the distance between these two points can not use the typical formula to find the distance between two points. This will affect our hypothesis test results "reject null hypothesis" or "fail to reject it". To better understand this question, we will use the following example. Let us assume the most common and elementary situation as the first course in elementary statistics. A random variable $\mathrm{X}$ is normally distributed with mean $\mu$ and variance $\sigma_{0}^{2}$, where $\mu$ is unknown to us but variance $\sigma_{0}^{2}$ is known. Also assume we wish to test the following hypothesis, $H_{0} \mu=\mu_{0}$ versus $\mathrm{H}_{\mathrm{a}} \mu \neq \mu_{0}$, with a sample of size one. We all know in this situation the critical region is $C=<\bar{x} /\left|\overline{\mathrm{x}}-\mu_{0}\right|>\mathrm{c} \sigma_{0}>$. Then, the question becomes, is "the distance between $N\left(\mu_{0}, \sigma_{0}^{2}\right)$ and $N\left(\bar{x}, \sigma_{0}^{2}\right)$ big enough for us to reject $H_{0}$ ?" Because it is not the usual straight line, and we know the Gaussian Curvature of the Normal distribution is a negative constant. So we should use distance function from hyperbolic surface of sheet to measure this distance.

\section{LIST THE FUNDAMENTAL TENSOR}

The probability density function for the lognormal Distribution is given by

$$
g(y, u, v)=\frac{1}{\sqrt{2 \pi} v y} \exp \left(-\frac{(\ln y-u)^{2}}{2 v^{2}}\right) \quad 0 \leq \mathrm{y} \leq \infty
$$

where $\mathrm{v}$ is the scale parameter and $\mathrm{u}$ is the shape parameter.

$$
\operatorname{lng}(\mathrm{y})=-(\ln \sqrt{2 \pi}+\ln v y)-\frac{(\ln y-u)^{2}}{2 v^{2}}
$$

From above equation, we derive the metric tensor components for the lognormal case as follow,

\footnotetext{
1 Corresponding Author:Williamuschen@gmail.com
} 


$$
\begin{aligned}
& E=-E\left(\frac{\partial^{2} \ln g(x)}{\partial u^{2}}\right)=\frac{1}{v^{2}}, \quad F=-E\left(\frac{\partial^{2} \ln g(x)}{\partial v \partial u}\right)=0, \\
& G=-E\left(\frac{\partial^{2} \ln g(x)}{\partial v^{2}}\right)=\frac{2}{v^{2}}
\end{aligned}
$$

$U \sin g$ above results, we can easily derive the following useful results

$$
\begin{aligned}
& \mathrm{E}_{\mathrm{u}}=0, \quad \mathrm{E}_{\mathrm{v}}=\frac{-2}{v^{3}}, \quad \mathrm{G}_{\mathrm{u}}=0, \quad \mathrm{G}_{\mathrm{v}}=\frac{-4}{v^{3}}, \quad \mathrm{EG}=\frac{2}{v^{4}} \\
& \Gamma_{11}^{1}=\frac{E_{u}}{2 E}=0, \quad \Gamma_{12}^{2}=\frac{G_{u}}{2 G}=0 \quad \Gamma_{11}^{2}=\frac{-E_{v}}{2 G}=\frac{1}{2 v}, \\
& \Gamma_{22}^{1}=\frac{-G_{u}}{2 E}=0 \quad \Gamma_{12}^{1}=\frac{E_{v}}{2 E}=\frac{-1}{v}, \Gamma_{22}^{2}=\frac{G_{v}}{2 G}=\frac{-1}{v}
\end{aligned}
$$

\section{THE GEODESIC EQUATION}

Method to find the geodesic equation of the lognormal distribution is by solving a triply of partial differential equations given in the appendix I. We seek its solution in the following section.

$$
\begin{aligned}
& \frac{d^{2} u}{d s^{2}}-\frac{2}{v} \frac{d u d v}{d s d s}=0, \\
& \frac{d^{2} v}{d s^{2}}+\frac{1}{2 v}\left(\frac{d u}{d s}\right)^{2}-\frac{1}{v}\left(\frac{d v}{d s}\right)^{2}=0,
\end{aligned}
$$

and the distance function

$$
\mathrm{ds}^{2}=\frac{1}{v^{2}} d u^{2}+\frac{2}{v^{2}} d v^{2}
$$

It only needs two out of above three equations to find lognormal model geodesic equation. We will choose the first and third equations. To simplify the notation, we let

$$
\mathrm{p}=\frac{\mathrm{du}}{\mathrm{ds}}, \text { then } \frac{\mathrm{dp}}{\mathrm{ds}}-\frac{2}{v} p \frac{d v}{d s}=0 .
$$

Divided above equation by $\mathrm{p}$, we get

$$
\frac{\frac{\mathrm{dp}}{\mathrm{ds}}}{\mathrm{p}}-\frac{2}{v} \frac{d v}{d s}=0 .
$$

Integration on both side w.r.t.p, we get

$$
\ln \mathrm{p}-2 \ln \mathrm{v}=\mathrm{C}_{1}
$$

$\ln \mathrm{pv}^{-2}=C_{1} \quad$ or $\quad \mathrm{pv}^{-2}=e^{C_{1}}=A$

so $\frac{\mathrm{du}}{\mathrm{ds}}=A v^{2} \quad$ Finally, we derive that

$$
\mathrm{ds}^{2}=\frac{d u^{2}}{A^{2} v^{4}}
$$


Substitute (3.4) into (3.3)

$$
\begin{aligned}
& \frac{d u^{2}}{A^{2} v^{4}}=\frac{1}{\mathrm{v}^{2}} d u^{2}+\frac{2}{v^{2}} d v^{2} \\
& \mathrm{du}^{2}=\mathrm{A}^{2} \mathrm{v}^{2}\left(\mathrm{du}^{2}+2 \mathrm{dv}^{2}\right) \\
& \left(1-\mathrm{A}^{2} \mathrm{v}^{2}\right) \mathrm{du}^{2}=2 \mathrm{~A}^{2} \mathrm{v}^{2} \mathrm{dv}^{2} \\
& \pm d u=\frac{ \pm \sqrt{2} A v d v}{\sqrt{1-\mathrm{A}^{2} v^{2}}}
\end{aligned}
$$

Integration both side, we get

$$
\begin{gathered}
\pm \mathrm{u}= \pm \int \frac{\sqrt{2} A v d v}{\sqrt{1-\mathrm{A}^{2} v^{2}}}+\mathrm{B} \\
\text { or } \pm \mathrm{u} \pm \int \frac{\sqrt{2} A v d v}{\sqrt{1-\mathrm{A}^{2} v^{2}}}=\mathrm{B}
\end{gathered}
$$

where $\mathrm{A}$ and $\mathrm{B}$ are arbitrary constant

Alternatively, we can find the geodesic equation of the lognormal distribution by solving one partial differential equation. This idea originated from French mathematician Darboux's theory. Detail proof has given in Chen [2][3]

From section 2, we know that the coefficient of the first fundamental form is given as,

$$
\mathrm{E}=\frac{1}{\mathrm{v}^{2}}, \quad \mathrm{~F}=0, \quad \mathrm{G}=\frac{2}{v^{2}} . \quad \text { or } \quad \mathrm{EG}-\mathrm{F}^{2}=\frac{2}{v^{4}}
$$

The equation $\nabla Z=1$ becomes

$$
\frac{1}{\mathrm{v}^{2}}\left(z_{v}^{2}+2 z_{u}^{2}\right)=\frac{2}{v^{4}}
$$

To solve above patial differential equation (3.5), we use the separable variable method as follow.

$$
\begin{aligned}
& z_{v}^{2}+2 z_{u}^{2}=\frac{2}{v^{2}} \text { or } \\
& 2 \mathrm{z}_{\mathrm{u}}^{2}=\frac{2}{v^{2}}\left(1-\frac{\mathrm{v}^{2}}{2} z_{v}^{2}\right) \text { hence, } \\
& \mathrm{z}_{\mathrm{u}}^{2}=\frac{1}{v^{2}}\left(1-\frac{\mathrm{v}^{2}}{2} z_{v}^{2}\right)=\mathrm{A}^{2} \quad \text { or } \\
& \mathrm{z}_{\mathrm{u}}= \pm A \quad \text { and } \quad \mathrm{z}= \pm \mathrm{Au}
\end{aligned}
$$

On the other hand, we also have

$$
\begin{aligned}
& \frac{1}{\mathrm{v}^{2}}\left(1-\frac{\mathrm{v}^{2}}{2} z_{v}^{2}\right)=\mathrm{A}^{2} \\
& \left(\frac{\partial z}{\partial v}\right)^{2}=\frac{2}{v^{2}}\left(1-A^{2} v^{2}\right) \\
& \mathrm{Z}= \pm \int \frac{\sqrt{2} \sqrt{1-A^{2} v^{2}}}{v} d v
\end{aligned}
$$

Combine solution (3.6) and (3.7), we finally find the general solution of lognormal Distribution $\mathrm{z}$ as follow. 


$$
z= \pm A u \pm \int \frac{\sqrt{2} \sqrt{1-A^{2} v^{2}}}{v} d v
$$

Thus, by applying the Darboux Theorem, we can find the geodesic equation of the lognormal

Distribution by taking partial derivative with respect to A and equal to B. i.e. $\frac{\partial \mathrm{z}}{\partial \mathrm{A}}=B$

$\pm u \pm \int \frac{\sqrt{2} A v d v}{\sqrt{1-A^{2} v^{2}}}=B$

As expected this solution coincide with theresult as method 1 result.

\section{APPENDIX I}

We list the six well known Christoffel Symbols as follows. For detail derivation see Struik[4] or Grey[5].

$$
\begin{array}{rlrl}
\Gamma_{11}^{1} & =\frac{G E_{u}-2 F F_{u}+F E_{v}}{2\left(E G-F^{2}\right)}, & \Gamma_{12}^{2} & =\frac{E G_{u}-F E_{v}}{2\left(E G-F^{2}\right)} \\
\Gamma_{11}^{2} & =\frac{2 E F_{u}-E E_{v}-F E_{u}}{2\left(E G-F^{2)}\right)}, & \Gamma_{22}^{1}=\frac{2 G F_{v}-G G_{u}-F G_{v}}{2\left(E G-F^{2}\right)} \\
\Gamma_{12}^{1}=\frac{G E_{v}-F G_{u}}{2\left(E G-F^{2}\right)}, & \Gamma_{22}^{2}=\frac{E G_{v}-2 F F_{v}+F G_{u}}{2\left(E G-F^{2}\right)}
\end{array}
$$

In general, the solution of the geodesic equation depends upon a pair of partial differential equations as below.

$$
\begin{aligned}
& \frac{d^{2} u}{d s^{2}}+\Gamma_{11}^{1}\left(\frac{d u}{d s}\right)^{2}+2 \Gamma_{12}^{1}\left(\frac{d u}{d s} \frac{d v}{d s}\right)+\Gamma_{22}^{1}\left(\frac{d v}{d s}\right)^{2}=0 \\
& \frac{d^{2} v}{d s^{2}}+\Gamma_{11}^{2}\left(\frac{d u}{d s}\right)^{2}+2 \Gamma_{12}^{2}\left(\frac{d u}{d s} \frac{d v}{d s}\right)+\Gamma_{22}^{2}\left(\frac{d v}{d s}\right)^{2}=0
\end{aligned}
$$

\section{APPENDIX II}

Statisticians have used the Bayesian theory to develop the so called "predictive distance". In a personal letter communication with Mitchell, A.F.S.[6][7] the author has learned the following example.

Consider the gamma distribution with known

$\gamma>0$ and $\theta=\lambda>0$, the estimative distance is

$\hat{d}_{M L}\left(\lambda_{1}, \lambda_{2}\right)=d\left(\hat{\lambda}_{1, M L}, \hat{\lambda}_{2, M L}\right)=\sqrt{\gamma}\left|\ln \left(\frac{t_{1}}{t_{2}}\right)\right|$

where $\mathrm{t}=\mathrm{m} \hat{\mathrm{x}}, \quad \hat{\lambda}_{\mathrm{ML}}=\frac{\gamma}{\tilde{x}}, \quad \mathrm{~g}(\lambda)=\frac{\gamma}{\lambda^{2}}$

Based on Jeffreys's Prior

$\pi(\lambda) \propto \sqrt{g(\lambda)} \propto \frac{1}{\lambda}$

the posterior density is

$\pi(\lambda \uparrow t)=\frac{\lambda^{m r-1} t^{m r} e^{-\lambda t}}{\Gamma(m r)} I_{(0, \infty)}(\lambda)$ 
The predictive distance then follows to be

$\hat{d}_{p}\left(\lambda_{1}, \lambda_{2}\right)=\sqrt{\frac{m r}{m r+r+1}} \hat{d}_{M L}\left(\hat{\lambda}_{1}, \hat{\lambda}_{2}\right)$

\section{REFERENCES}

[1] Chen W.W.S. (2013) On computing Gaussian Curvature of some well known distribution. Theoretical Mathematics \& Applications, Volume 3, issue 4, 2013.

[2] Chen W.W.S. (2014) A Note On Finding Geodesic Equation of two Parameters Gamma Distribution. Applied Mathematics, www.scirp.org/journal/am ,5, 3511-3517.

[3] Chen W.W.S. (2014) A note on finding geodesic equation of two parameter Weibull distribution. Theoretical Mathematics \& Applications, Vol.4, no. 3, 43-52.

[4] Struik, D.J.(1961) Lectures on Classical Differential Geometry. Second Edition. Dover Publications,Inc.

[5] Grey, A.(1993) Modern Differential Geometry of Curves and Surfaces. CRC Press, Inc. Boca Raton.

[6] Mitchell, A.F.S.(1992) Estimative and predictive distances. Test, 1, 105-21.

[7] Mitchell, A.F.S. and Krzanowski, W.J.(1985) The Mahalanobis distance and elliptic distributions. Biometrika, 72, 464-7. 